\title{
Combined Microsurgery and Radiotherapy for Multiple Spinal Cord Hemangioblastomas with Holocord Syrinx in von Hippel-Lindau Disease: A Case Report
}

\author{
Nicolas Knoop $^{1}$ Clemens Seidel $^{2}$ Clara Frydrychowicz $^{3}$ Jürgen Meixensberger ${ }^{1}$
}

${ }^{1}$ Department of Neurosurgery, University of Leipzig, Leipzig, Germany

2 Department of Radiotherapy, University of Leipzig, Leipzig, Germany

Address for correspondence Nicolas Knoop, MD, Department of

${ }^{3}$ Department of Neuropathology, University of Leipzig, Leipzig, Germany Neurosurgery, University of Leipzig, Liebigstraße 20, 04103 Leipzig, Germany (e-mail: nicolas.knoop@medizin.uni-leipzig.de).

J Neurol Surg Rep 2019;80:e46-e50.

\author{
Abstract \\ Keywords \\ - hemangioblastoma \\ - von Hippel-Lindau \\ - microsurgery \\ - radiotherapy \\ - syringomyelia \\ - holocord syrinx
}

Spinal and cerebellar hemangioblastomas are common in von Hippel-Lindau disease (vHLD) and usually treated surgically. Multifocal presence and surgically not amenable locations are issues that require a combined microsurgical and radiosurgical approach to control complex cases.

We would like to present the case of a 37-year-old male patient who was diagnosed vHLD with multiple spinal and one infratentorial hemangioblastomas and holocord syrinx formation of the whole spinal cord. Combined microsurgical approaches to two spinal lesions and the cerebellar lesion followed by external beam radiotherapy of the posterior fossa and the whole spinal axis stabilized tumor growth of the asymptomatic lesions, while no recurrent tumors were detected at the site of surgery. A clinical deterioration connected to early postoperative deficits stabilized to a moderate gait ataxia. The follow-up after radiotherapy covered 60 months.

A combination of microsurgery and radiosurgery for the surgically not amenable lesions is an adequate treatment regimen to stabilize tumor growth and clinical symptoms of multifocal spinal hemangioblastomas in vHLD, though the therapy should be limited to symptomatic or growing lesions.

\section{Introduction}

von Hippel-Lindau disease (vHLD) constitutes an autosomal dominantly inherited multisystem neoplasia syndrome due to a mutation on the short arm of chromosome 3 (3p25). Typical manifestations are angiomas of the retina (central serous retinitis) and hemangioblastomas (HABs) of the infratentorial and spinal central nervous system (CNS) region (predominantly posterior fossa). Further neoplasias are renal cell carcinomas, pancreatic neuroendocrine tumors, pheochromocytomas, and endolymphatic sac tumors. ${ }^{1-3}$

Though rarely encountered sporadically, spinal HABs are common in vHLD as multifocal lesions that often require multiple surgical interventions. We present a case of vHLD with complicated multiple spinal HABs and holocord syrinx formation undergoing both microsurgery and radiotherapy in a short follow-up period. In current literature, radiotherapy for spinal HABs is reviewed and outcomes are discussed with regard to the presented case.

\section{Clinical Presentation}

Our 37-year-old male patient had ophthalmologic examination due to progressive visual disturbance. Fundoscopy revealed central serous retinitis. Due to positive family history for renal cell carcinoma in the patient's mother, more extensive diagnostics were performed. Cranial and received

April 18, 2019

accepted

September 28, 2019
DOI https://doi.org/

10.1055/s-0039-3401808. ISSN 2193-6358. (c) 2019 Georg Thieme Verlag KG
Stuttgart · New York

License terms

c) $(1) \$$ 

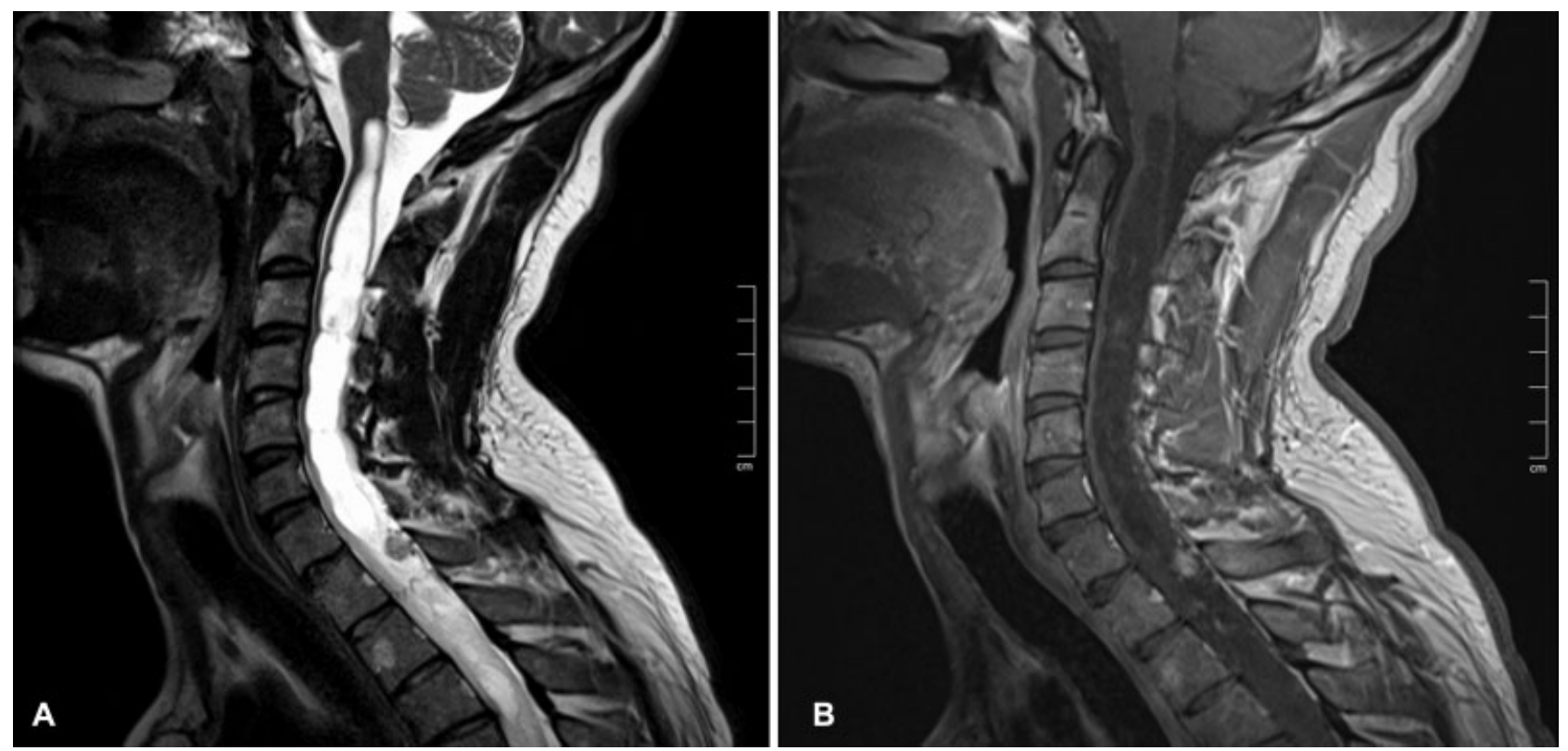

Fig. 1 Magnetic resonance imaging scans of the cervical spine on first clinical onset of symptoms, T2-weighted (A), and contrast-enhanced T1weighted (B) images showing an extended syrinx formation due to a large contrast enhancing formation at the cervicothoracic junction.

whole spine magnetic resonance imaging (MRI) showed holocord syrinx formation with multiple contrast enhancing nodular lesions, predominantly in the right cerebellar tonsil and at spinal cord levels $\mathrm{T} 1, \mathrm{~T} 2$, and at $\mathrm{T} 11 / 12$ junction that were consistent with HABs (-Figs. 1 and 2). On neurological examination, he presented with increased lower limb reflex levels, mild gait ataxia, and a tendency to fall to the right. All of these signs were not previously noticed by the patient. vHLD was genetically proven in the patient and his mother.
He first underwent laminectomies C7 and T1 to approach the upper lesions and decompress the syrinx. Intraoperatively, only one lesion at T1 could safely be removed and the syrinx was fenestrated.

Histology showed a tumor of moderate cell density. The tumor was composed of large stromal cells with either vacuolated or homogeneously eosinophilic cytoplasm. These were embedded in a dense capillary network. Stroma cells stained positive for S100; the capillary network was
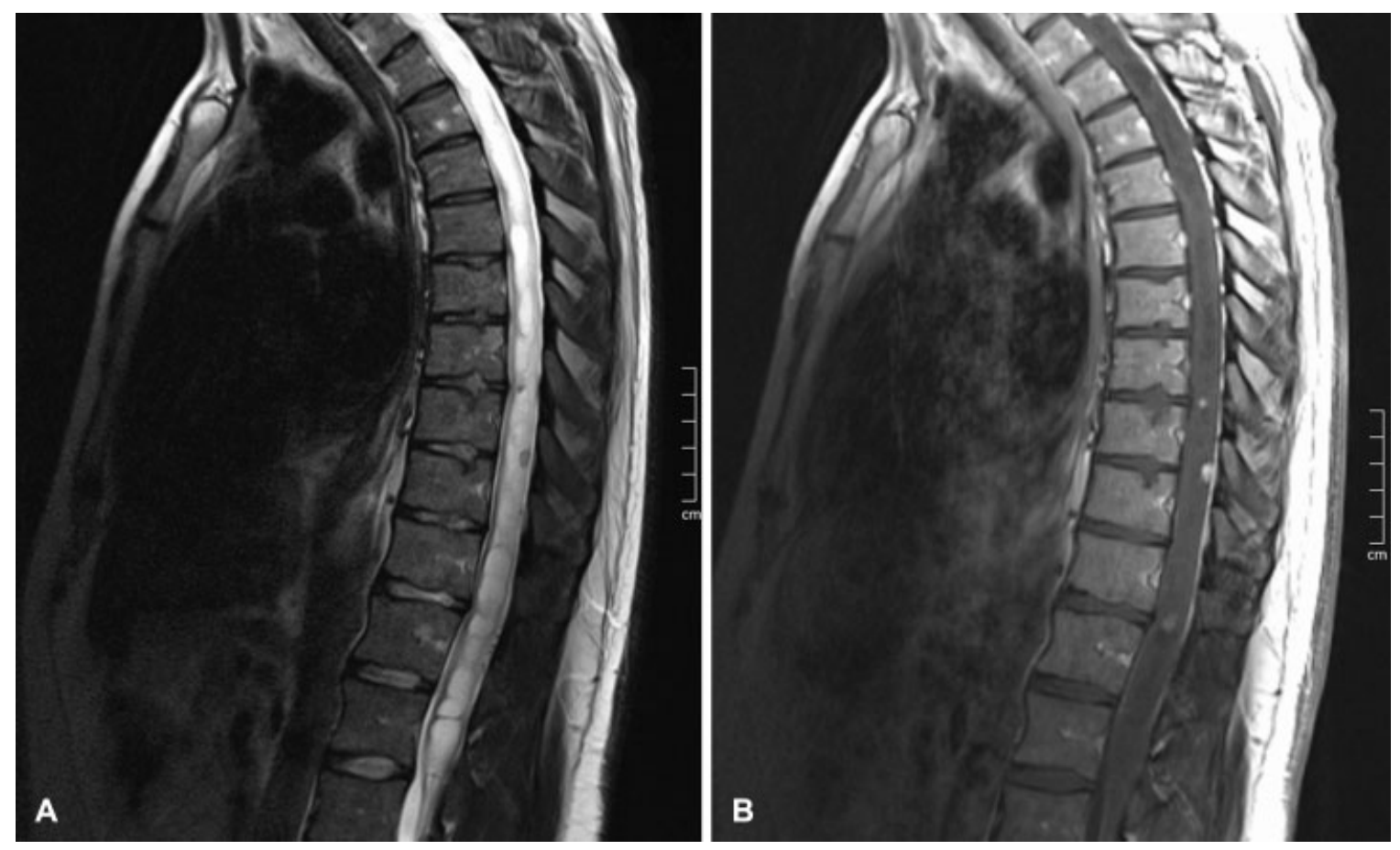

Fig. 2 Magnetic resonance imaging scans of the thoracic spine on first clinical onset of symptoms. T2-weighted (A) and contrast enhanced T1weighted (B) images further demonstrate the extent of the syrinx down to the conus medullaris among multiple contrast enhancing lesions. 
highlighted by Tibor Pap silver impregnation and factor VIII immunohistochemistry. Proliferation index was below $2 \%$. Histomorphology and immunohistochemical staining pattern were consistent with the diagnosis of HAB WHO $\mathrm{I}^{\circ}$. vHLD-associated HABs are histomorphologically indistinguishable from sporadic HABs.

The lesion at $\mathrm{T} 2$ was left untouched due to total adherence to the corresponding nerve root. Histological diagnosis was HAB. The postoperative course was uneventful except of temporary left hemi hypoesthesia sub-Th5. Postoperative MRI showed a collapse of the cervical portion of the syrinx and total resection of the lesion at T1. Early MRI controls revealed progression of the cervical portion of the syrinx. Hence, another surgical procedure via laminectomy T12 was performed to resect the lesion at $\mathrm{T} 11 / 12$ junction, but the resection was not possible for a too anterior position of the vascular lesion. Additionally, a syringo-subdural shunt was implanted. Postoperatively the patient presented with isolated ataxia of the right leg.

After scooping out the options for surgical treatment, radiotherapy of the spinal cord was planned, but before initiating the latter, control MRI showed progression of the accompanying cyst of the right cerebellar tonsil lesion. The clinical presentation did not change, while the right leg ataxia was still present. So, the patient first underwent suboccipital craniotomy. The lesion could be totally removed.

Hence, irradiation of the posterior fossa and the spinal axis was performed using conventional computed tomographyplanned isocentric multifield external beam radiotherapy with $5 \times 1.8$ Gy weekly until 36 Gy. Sequentially, a boost of $9 \mathrm{~Gy}$ to the tumor bed in the posterior fossa and to the multiple spinal hemangiomas from $\mathrm{C} 7$ to Th12 was performed up to a total dose of $45 \mathrm{~Gy}$.

The patient afterward developed lower limb weakness and progressive gait ataxia requiring the use of corticosteroids. The control MRI 2 months after radiotherapy showed a progression of one remaining lesion at T2 accompanied by an extension of the cervicothoracic portion of the syrinx. Thus, a second approach to the lesion was undergone via laminectomy $\mathrm{T} 2$ and the lesion could be totally resected.

Throughout the further clinical course after the last surgery, a mild improvement in the gait ataxia was noticeable. Followup MRIs demonstrated a remarkable collapse of the cervical portion of the syrinx and a stable size of the remaining multiple nodular intramedullary lesions (-Figs. 3 and $\mathbf{4}$ ). The whole follow-up period after radiotherapy covered 60 months.

During this period, the patient underwent multiple ophthalmologic surgeries and intravitreal local radiotherapy for a HAB of the left retina. He also underwent partial left sided nephrectomy due to renal cell carcinoma.

Overall, the patient's neurologic status was fluctuating deterioration linked to the surgical procedures but stabilized to a moderate gait ataxia and hyperreflexia of the lower limbs.

\section{Discussion}

Spinal cord HABs account for a relatively small amount of primary spinal cord tumors. ${ }^{4}$ Although they are often accompanied by local syringomyelia, only a few cases with an extensive, holocord syrinx have been published to date. ${ }^{5-7}$ Since the signs and symptoms in patients with HABs mostly arise from peritumoral cysts and syrinx formation, treatment strategies should focus not only on curative but also on good functional outcome. Hence, the resolution of local and more extensive mass effects, such as syringomyelia, is a definitive target of treatment. ${ }^{2,3}$ The judicious treatment of symptom producing lesions while avoiding unnecessary treatment of asymptomatic tumors, which might not grow, could, according to Lonser et al, provide clinical stability. ${ }^{8}$

Although surgical resection constitutes the therapy of choice for spinal HABs, radiotherapy has become increasingly
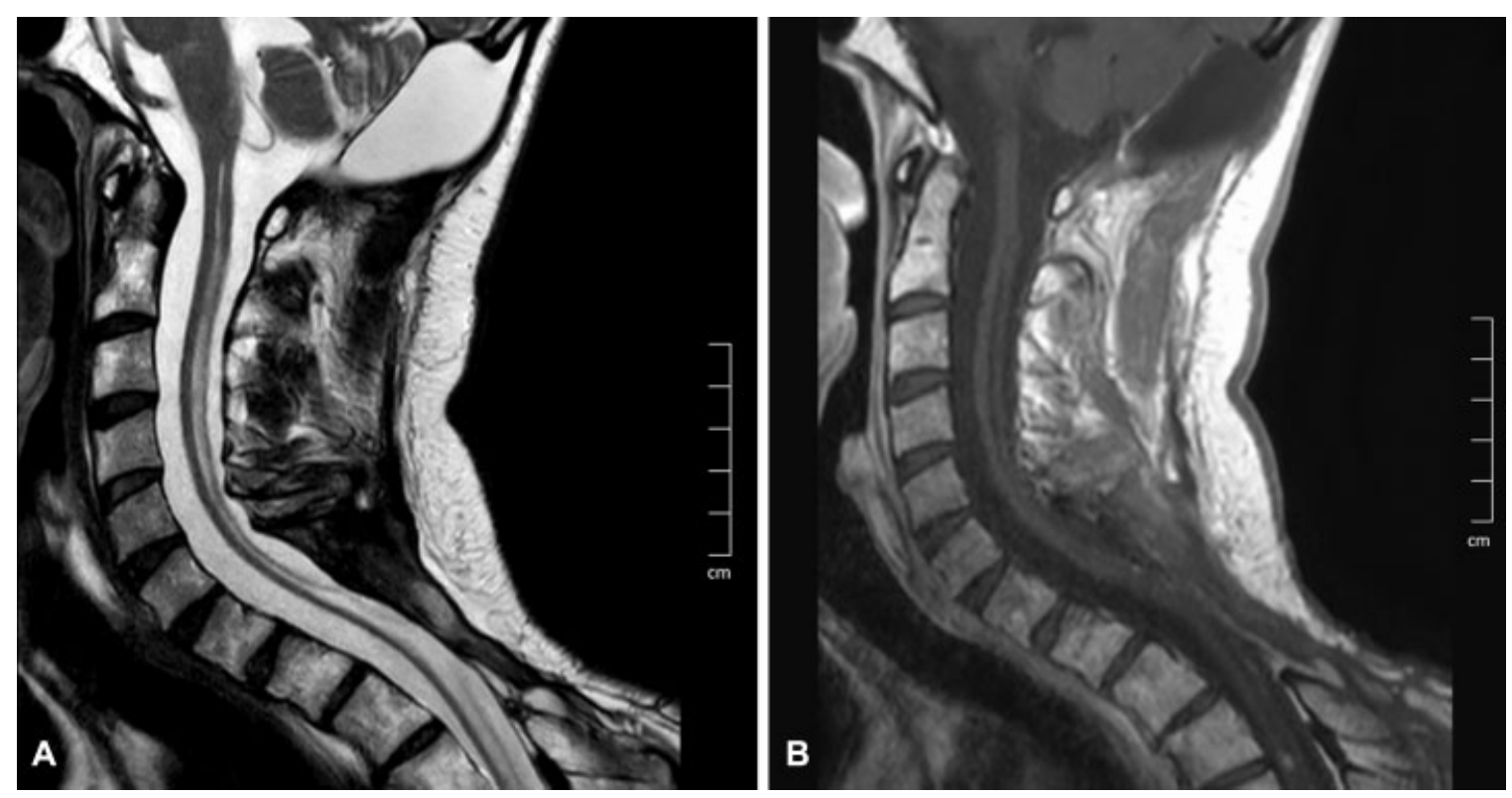

Fig. 3 Magnetic resonance imaging scans of the cervical spine 60 months after radiotherapy. T2-weighted (A) and contrast-enhanced T1-weighted (B) images demonstrate a considerable collapse of the cervical portion of the syrinx after resection of the cervicothoracic hemangioblastoma. 


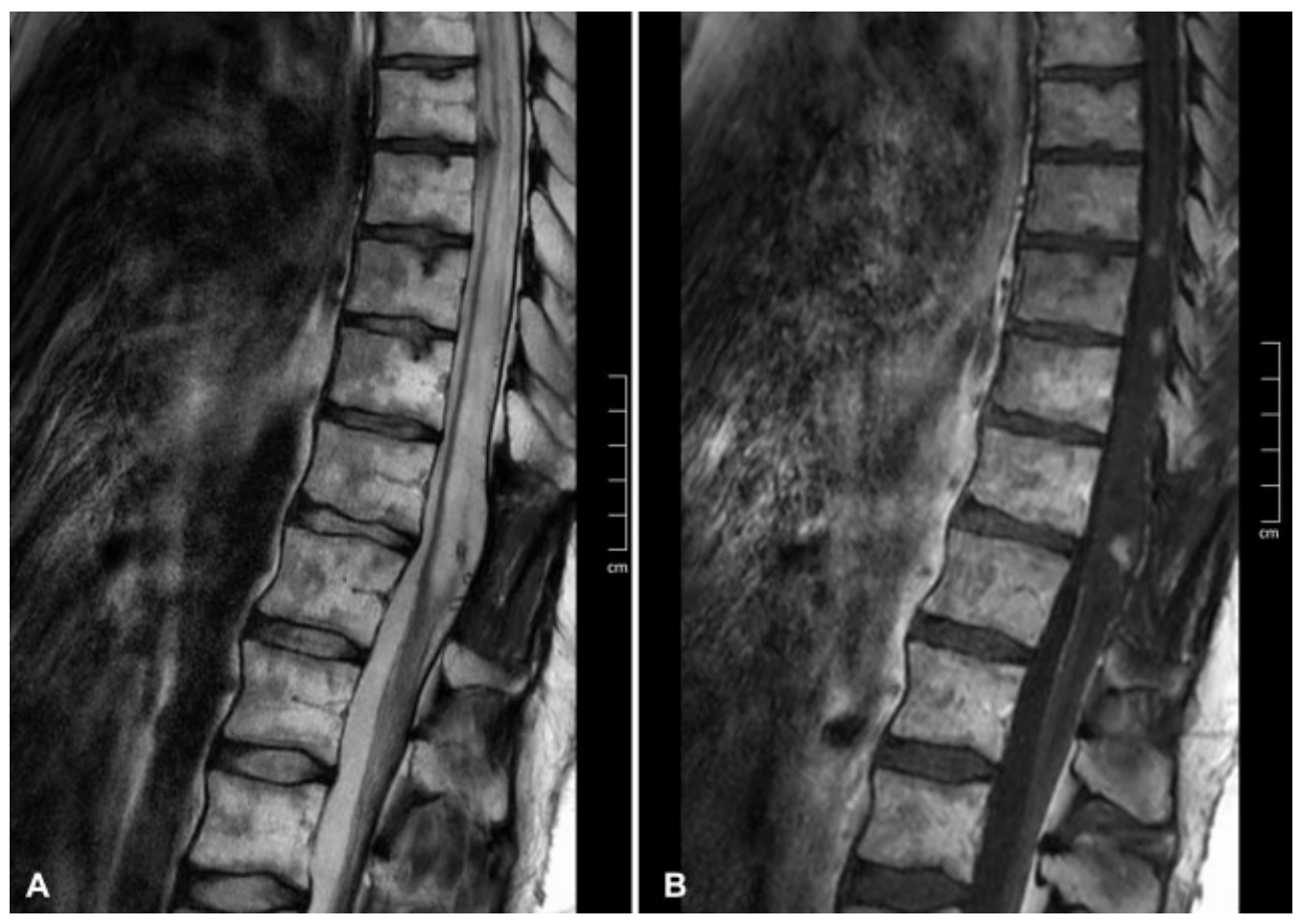

Fig. 4 Magnetic resonance imaging scans of the thoracic spine 60 months after radiotherapy. T2-weighted (A) and contrast-enhanced T1weighted (B) images show partial collapse of the thoracic portion of the syrinx and stable-sized contrast enhancing lesions after radiotherapy.

important for the treatment of surgically not amenable lesions in both cranial and spinal locations. ${ }^{8-22}$

As published by various authors before, high-dose focal radiotherapy for HABs can achieve stabilization of tumor growth (-Table $\mathbf{1}$ ) in the per se not particularly radiosensitive tumors.

However, results of studies with radiotherapy for HAB have to be interpreted with caution as the known multiphasic growth pattern of these tumors makes interpretation of tumor size during follow-up difficult; therapeutic effects and intrinsic changes of tumor size can possibly not be distinguished; and long-term follow-up is needed to better evaluate efficacy of treatment. As shown by Asthagiri et al for intracranial HABs, excellent local control rates (> 80\%) 2 years after SRS are diminishing during long-term analysis (68\%, control rate after 8.5 years). ${ }^{23}$

For interpretation of clinical data, it is relevant that HABs in patients with vHLD might have better overall survival and longer disease-free intervals compared with sporadic tumors. $^{24}$

Radiotherapy of multifocal spinal HAB of our case report remains a particular therapeutic challenge: (1) identification of "first to treat" symptomatic lesions might be difficult, (2) the decision for or against treatment of asymptomatic lesions

Table 1 Previous studies on radiotherapy for spinal hemangioblastomas

\begin{tabular}{|l|l|l|l|l|l|}
\hline Author & Method & $\begin{array}{l}\text { Patients with } \\
\text { spinal HAB }\end{array}$ & Spinal HABs & $\begin{array}{l}\text { Mean follow-up } \\
\text { (months) }\end{array}$ & Outcome \\
\hline Chang et al., $1998^{16}$ & SRS & 2 & 2 & 13.5 & Tumor decreased \\
\hline Koh et al., $2007^{24}$ & EBRT & 18 & 8 & 61 & 5-year disease free survival, 57\% \\
\hline Ryu et al., $2003^{17}$ & SRS & 7 & 7 & 12 & Local control rate, $100 \%$ \\
\hline Moss et al., $2009^{25}$ & SRS & 31 & 16 & 33.5 & Local control rate, 92\% \\
\hline Chang et al., $2011^{15}$ & SRS & 5 & 8 & 50 & Local control rate, $87.5 \%$ \\
\hline Simone et al., $2011^{20}$ & ICSRT & 7 & $\begin{array}{l}84 \text { (spinal and } \\
\text { infratentorial) }\end{array}$ & 73.8 & Local control rate, $24 \%$ \\
\hline Selch et al., $2012^{21}$ & LINAC & 9 & 20 & 51 & Local control rate, 95\% \\
\hline Pan et al., $2017^{22}$ & SRS & 28 & 46 & 54 & Local control rate, $94 \%$ \\
\hline
\end{tabular}

Abbreviations: EBRT, external beam radiotherapy; ICSRT, infratentorial craniospinal radiation therapy; LINAC, linear accelerator; SRS, stereotactic radiosurgery. 
is controversial due to unpredictable growth, (3) dose constraints of the spinal cord restrict use of conventional radiotherapy, (4) nearly half of the vHLD-associated HABs is not visible on the first imaging series. ${ }^{3}$

To improve prognosis in this complicated setting, infratentorial craniospinal irradiation has been used as a treatment approach. Simone et al first described a case series of this technique using conventional multifield infratentorial craniospinal irradiation $(24 \times 1.8 \mathrm{~Gy}, 43.2 \mathrm{~Gy})$ to the entire craniospinal region without the hemispheres in seven patients with diffuse and multilocal CNS HABs with vHLD. Although local control was limited (23.9\%) and the development of new lesions could not be limited, clinical stabilization or even improvement in five of seven patients during a mean follow-up period of $\sim 74$ months could point toward some efficacy of this approach. ${ }^{20}$

\section{Conclusion}

In conclusion, treatment of multiple HABs requires a multidisciplinary approach, optimization of treatment is made difficult by growth pattern of tumors and long-term followup on radiological control, and functional outcome is needed to evaluate and improve quality of treatment.

Conflict of Interest

None.

\section{References}

1 Maher ER, Yates JR, Harries R, et al. Clinical features and natural history of von Hippel-Lindau disease. Q J Med 1990;77(283): 1151-1163

2 Wanebo JE, Lonser RR, Glenn GM, Oldfield EH. The natural history of hemangioblastomas of the central nervous system in patients with von Hippel-Lindau disease. J Neurosurg 2003;98 (01):82-94

3 Ammerman JM, Lonser RR, Dambrosia J, Butman JA, Oldfield EH. Long-term natural history of hemangioblastomas in patients with von Hippel-Lindau disease: implications for treatment. J Neurosurg 2006;105(02):248-255

4 Miller DJ, McCutcheon IE. Hemangioblastomas and other uncommon intramedullary tumors. J Neurooncol 2000;47(03):253-270

5 Pai SB, Krishna KN. Secondary holocord syringomyelia with spinal hemangioblastoma: a report of two cases. Neurol India 2003;51 (01):67-68

6 Wu T-C, Guo W-Y, Lirng J-F, et al. Spinal cord hemangioblastoma with extensive syringomyelia. J Chin Med Assoc 2005;68(01): 40-44

7 Borkar SA, Kasliwal MK, Suri A, Sharma BS. Cervical hemangioblastoma with holocord syrinx. Surg Neurol 2009;72(04):437-438

8 Lonser RR, Weil RJ, Wanebo JE, DeVroom HL, Oldfield EH. Surgical management of spinal cord hemangioblastomas in patients with von Hippel-Lindau disease. J Neurosurg 2003;98(01):106-116
9 Murota T, Symon L. Surgical management of hemangioblastoma of the spinal cord: a report of 18 cases. Neurosurgery 1989;25 (05):699-707, discussion 708

10 Mandigo CE, Ogden AT, Angevine PD, McCormick PC. Operative management of spinal hemangioblastoma. Neurosurgery 2009; 65(06):1166-1177

11 Mehta GU, Asthagiri AR, Bakhtian KD, Auh S, Oldfield EH, Lonser RR. Functional outcome after resection of spinal cord hemangioblastomas associated with von Hippel-Lindau disease. J Neurosurg Spine 2010;12(03):233-242

12 Kim TY, Yoon DH, Shin HC, et al. Spinal cord hemangioblastomas in von Hippel-Lindau disease: management of asymptomatic and symptomatic tumors. Yonsei Med J 2012;53(06):1073-1080

13 Richardson RG, Griffin TW, Parker RG. Intramedullary hemangioblastoma of the spinal cord: definitive management with irradiation. Cancer 1980;45(01):49-50

14 Smalley SR, Schomberg PJ, Earle JD, Laws ER Jr, Scheithauer BW, O'Fallon JR. Radiotherapeutic considerations in the treatment of hemangioblastomas of the central nervous system. Int J Radiat Oncol Biol Phys 1990;18(05):1165-1171

15 Chang U-K, Rhee CH, Youn SM, Lee DH, Park SQ. Radiosurgery using the Cyberknife for benign spinal tumors: Korea Cancer Center Hospital experience. J Neurooncol 2011;101(01):91-99

16 Chang SD, Meisel JA, Hancock SL, Martin DP, McManus M, Adler JR Jr. Treatment of hemangioblastomas in von Hippel-Lindau disease with linear accelerator-based radiosurgery. Neurosurgery 1998; 43(01):28-34, discussion 34-35

17 Ryu SI, Kim DH, Chang SD. Stereotactic radiosurgery for hemangiomas and ependymomas of the spinal cord. Neurosurg Focus 2003;15(05):E10

18 Rajaraman C, Rowe JG, Walton L, Malik I, Radatz M, Kemeny AA. Treatment options for von Hippel-Lindau's haemangioblastomatosis: the role of gamma knife stereotactic radiosurgery. Br J Neurosurg 2004;18(04):338-342

19 Wang E-M, Pan L, Wang B-J, et al. The long-term results of gamma knife radiosurgery for hemangioblastomas of the brain. J Neurosurg 2005;102(Suppl):225-229

20 Simone CB II, Lonser RR, Ondos J, Oldfield EH, Camphausen K, Simone NL. Infratentorial craniospinal irradiation for von HippelLindau: a retrospective study supporting a new treatment for patients with CNS hemangioblastomas. Neuro-oncol 2011;13 (09):1030-1036

21 Selch MT, Tenn S, Agazaryan N, Lee SP, Gorgulho A, De Salles AA. Image-guided linear accelerator-based spinal radiosurgery for hemangioblastoma. Surg Neurol Int 2012;3:73

22 Pan J, Ho AL, D'Astous M, et al. Image-guided stereotactic radiosurgery for treatment of spinal hemangioblastoma. Neurosurg Focus 2017;42(01):E12

23 Asthagiri AR, Mehta GU, Zach L, et al. Prospective evaluation of radiosurgery for hemangioblastomas in von Hippel-Lindau disease. Neuro-oncol 2010;12(01):80-86

24 Koh E-S, Nichol A, Millar B-A, Ménard C, Pond G, Laperriere NJ. Role of fractionated external beam radiotherapy in hemangioblastoma of the central nervous system. Int J Radiat Oncol Biol Phys 2007;69(05):1521-1526

25 Moss JM, Choi CYH, Adler JR, Soltys SG, Gibbs IC, Chang SD. Stereotactic radiosurgical treatment of cranial and spinal hemangioblastomas. Neurosurgery 2009;65(01):75-85 